\title{
Interpenetrating Polymer Networks Based on Modified Castor Oil Urethane and Poly(methyl methacrylate)
}

\author{
Vilas AthaWALE ${ }^{\dagger}$ and Suresh KoleKar \\ University of Mumbai, Department of Chemistry, \\ Vidyanagari, Santacruz (E), Mumbai 400 098, India
}

(Received February 12, 1998)

\begin{abstract}
Castor oil was initially subjected to interesterification reaction with linseed oil, and the resulting intermediate was used for the preparation of urethane and their interpenetrating polymer networks (IPNs). The homopolymers and IPNs were characterized for physico-mechanical and thermal properties. The morphologies of IPNs were studied by scanning electron microscopy, and differential scanning calorimetry. On comparing the mechanical properties of unmodified and modified castor oil urethane and their IPNs it was found that modified castor oil (with $30 \mathrm{wt} \%$ linseed oil) showed higher tensile strength and hardness and lower elongation. IPNs of modified castor oil urethane and poly(methyl methacrylate) (PMMA) showed better compatibility than unmodified castor oil urethane/PMMA IPNs. No significant change was observed in case of thermal behavior. KEY WORDS Interesterification / Poly(methyl methacrylate) / Urethane / Interpenetrating Polymer Networks /
\end{abstract}

Castor oil is a commercially important natural product containing unsaturation and hydroxyl functionality. It is a renewable resource material abundantly available in India. It could be used to make new polymeric material meeting the expectations of polymer industries. The hydroxyl functionality of castor oil has been widely used to make crosslinked polyester and polyurethane. In recent years, attention has been focused on interpenetrating polymer networks (IPNs) based on castor oil. These materials are defined as a combination of two network polymers, at least one of which is prepared/crosslinked in the immediate presence of the other. Conversion of castor oil into its urethane prepolymer and then sequential or simultaneous interpenetration of vinyl monomers into the urethane network to get high strength polymers was studied and reported by many authors. ${ }^{1-8}$ Although, a wealth of literature is available on polyurethane prepared from modified castor oil, ${ }^{9-12}$ very few reports are available on utilization in IPNs.

IPNs synthesized from castor oil polyurethane and poly(methyl methacrylate) (PMMA) though elastomeric in nature are far from plastic. In order to explore the best combination of castor oil urethane-PMMA IPNs towards their application as a reinforced plastic rather than mere elastomeric materials, in the present work, we synthesized and characterized the IPNs of the desired properties from the modified castor oil and PMMA.

\section{EXPERIMENTAL}

\section{Materials}

British Standard Specifications (BSS) grade castor oil was procured from M/s Jayant Oil Mill (India), Isophorone diisocyanate (IPDI) and benzoyl peroxide were obtained from Fluka (switzerland) and Thomas Baker Co. (India), respectively. Methyl methacrylate (MMA) and ethylene glycol dimethacrylate (EGDMA) purchased from Sisco-Chem (India) and Fluka (Switzerland) were washed with $2 \% \mathrm{NaOH}$, dried over $\mathrm{CaCl}_{2}$ and distilled

\footnotetext{
† To whom correspondence should be addressed.
}

under reduced pressure. Dibutyltin dilaurate (DBTL), Fascat 4100 (tin catalyst for interesterification) and alkali refined linseed oil were obtained from local market. All other reagents were of analytical grade and have been used without further purification.

\section{Synthesis (Scheme 1)}

Synthesis of Castor Oil-Linseed Oil Interesterification Intermediate (ICL). Castor oil and linseed oil, in given proportions were taken into a reaction kettle equipped with thermometer, water condenser, stirrer and nitrogen inlet. The mixture was heated to $200^{\circ} \mathrm{C}$ and Fascat 4100 catalyst $(0.05 \mathrm{wt} \%$ based on castor oil) was added. The temperature was raised to $230^{\circ} \mathrm{C}$ and maintained between 230 to $235^{\circ} \mathrm{C}$, with constant stirring in an atmosphere of nitrogen for $2 \mathrm{~h}$. The reaction was monitored by thin layer chromatography (solvent system: petroleum ether, diethyl ether, and acetic acid in a ratio of $85: 15: 1$ by volume, respectively). In the later stage traces of water in the resin were removed by adding little amount of xylene to the kettle and distilling it under vacuum. Hydroxyl and acid values of the resulting product were determined $^{13}$ and appear in Table I.

Synthesis of Urethane Network $(U)$. A reaction kettle equipped with stirrer and nitrogen inlet, was charged with one equivalent of $50 \%$ solution of castor oil-linseed oil interesterified product in dioxane, followed by the addition of two equivalent of IPDI along with catalyst DBTL $\left(0.01 \mathrm{~g} \mathrm{~g}^{-1}\right.$ of IPDI) and was kept in a thermostated water bath. The temperature was then raised to $40 \pm 2{ }^{\circ} \mathrm{C}$ and maintained for $2 \mathrm{~h}$ with continuous stirring. After completion of $2 \mathrm{~h}$, triethanolamine (1.0 $\mathrm{wt} \%$ of the resin for chain extension and curing) was added and mixed thoroughly for $5 \mathrm{~min}$. The solution was then poured into the mold, where it was kept initially at $78^{\circ} \mathrm{C}$ for $24 \mathrm{~h}$ and then at $120^{\circ} \mathrm{C}$ for $4 \mathrm{~h}$.

Interpenetrating Polymer Networks (IPNs). The reaction kettle containing urethane prepolymer was charged with triethanolamine ( $1.0 \mathrm{wt} \%$ based on urethane), predetermined amount of MMA along with EGDMA (1.0 $\mathrm{wt} \%$ based on MMA), and benzoyl peroxide $(0.5 \mathrm{wt} \%$ 


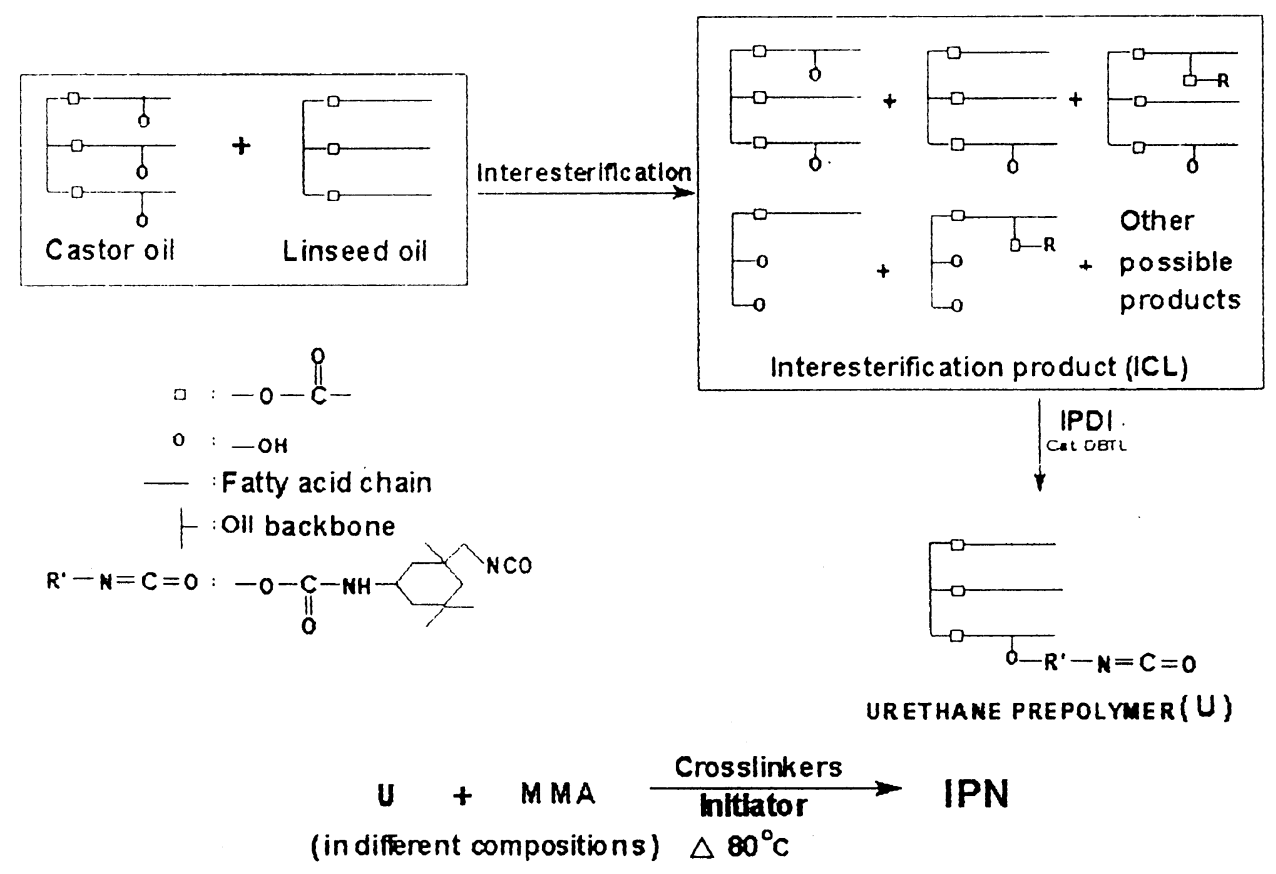

Scheme 1. The synthesis of modified castor oil urethane and IPNs with PMMA.

Table I. Acid and hydroxyl values for ICL intermediates in $\mathrm{mg} \mathrm{KOH} \mathrm{g}^{-1}$ of sample

\begin{tabular}{lccc}
\hline \multicolumn{4}{c}{ ICL intermediates based on ${ }^{\mathrm{a}}$} \\
\hline Property & $80 \% \mathrm{CO}$ & $70 \% \mathrm{CO}$ & $100 \% \mathrm{CO}$ \\
& $20 \% \mathrm{LO}$ & $30 \% \mathrm{LO}$ & \\
Acid value & 3.00 & 3.4 & 3.3 \\
Hydroxyl value & 143.47 & 133.18 & 162 \\
\hline
\end{tabular}

CO: Castor oil. LO: Linseed oil. a Composition based on total weight of the mixture in $\mathrm{wt}^{\mathrm{o}} \mathrm{o}$.

based on MMA) were intimately mixed for $3 \mathrm{~min}$ at $40^{\circ} \mathrm{C}$. The temperature was raised to 75 to $78^{\circ} \mathrm{C}$ and the reaction was continued for $60 \mathrm{~min}$ and then poured into the mold and cured initially at 75 to $78^{\circ} \mathrm{C}$ for $24 \mathrm{~h}$ and later at $120^{\circ} \mathrm{C}$ for $6 \mathrm{~h}$.

\section{Characterizations}

Infrared spectra of the IPNs were obtained from Shimadzu FTIR 4200 series spectrophotometer using $\mathrm{KBr}$ pellets, whereas, in case of urethane prepolymer, being of thick syrupy nature, a thin film was cast over $\mathrm{NaCl}$ block. Tensile strength and elongation at break were measured using computerized tensile testing machine "Tensilon" (R \& D Electronics, India) as per ASTM D 638, and hardness was determined with Shore A durometer using ASTM D 2240-75 standard. The apparent density of the films was obtained by weighing strips of film of measured volume. ${ }^{14}$ A swelling study was carried out in water, methyl ethyl ketone (MEK), and toluene. ${ }^{15}$ Thermogravimetric measurements were made on a Mettler TA 4000 thermogravimetric analyzer (TGA) at a heating rate of $10^{\circ} \mathrm{Cmin}^{-1}$ in nitrogen atmosphere and energy of activation was computed by using Broido method. ${ }^{16}$ Sample morphology was studied by scanning electron microscopy and the glass transition temperatures were determined by differential scanning calorimetry on Du Pont 9900 thermal analyzer at a heating rate of $10^{\circ} \mathrm{C} \mathrm{min}^{-1}$ in nitrogen atmosphere.
Table II. Mechanical properties of hompolymers and IPNs

\begin{tabular}{|c|c|c|c|c|}
\hline \multirow{2}{*}{$\begin{array}{l}\text { Sample } \\
\text { code }\end{array}$} & $\begin{array}{c}\text { Composition } \\
\text { U/PMMA }\end{array}$ & $\begin{array}{l}\text { Tensile } \\
\text { strength }\end{array}$ & $\begin{array}{l}\text { Elongation } \\
\text { at break }\end{array}$ & \multirow{2}{*}{$\begin{array}{c}\text { Hardnesss } \\
\text { Shore A }\end{array}$} \\
\hline & $w t \%$ & $\mathrm{MPa}$ & $\%$ & \\
\hline $\mathrm{CU}^{\mathrm{a}}$ & $100 / 0$ & 0.85 & 142 & 72 \\
\hline IPN-1 & $25 / 75$ & 1.91 & 48 & 92 \\
\hline IPN-2 & $50 / 50$ & 1.63 & 242 & 76 \\
\hline IPN-3 & $75 / 25$ & 1.02 & 310 & 71 \\
\hline $\mathrm{ICL1U}^{\mathrm{b}}$ & $100 / 0$ & 1.25 & 128 & 81 \\
\hline IPN-4 & $25 / 75$ & 0.78 & 8 & 98 \\
\hline IPN-5 & $50 / 50$ & 0.65 & 37 & 96 \\
\hline IPN-6 & $75 / 25$ & 0.54 & 103 & 88 \\
\hline $\mathrm{ICL}_{2} \mathrm{U}^{\mathrm{c}}$ & $100 / 0$ & 1.44 & 97 & 87 \\
\hline IPN-7 & $25 / 75$ & 2.18 & 10 & $>100$ \\
\hline IPN-8 & $50 / 50$ & 2.06 & 91 & 100 \\
\hline IPN-9 & $75 / 25$ & 1.57 & 188 & 92 \\
\hline PMMA & $0 / 100$ & 62.00 & 8 & Hard \\
\hline
\end{tabular}

${ }^{\mathrm{a}} \mathrm{CU}=$ unmodified castor oil urethane. ${ }^{\mathrm{b}} \mathrm{ICL} 1 \mathrm{U}=$ modified castor oil (using $20 \%$ linseed oil) urethane. ${ }^{\mathrm{c}} \mathrm{ICL} 2 \mathrm{U}=$ modified castor oil (using $30 \%$ linseed oil) urethane.

\section{RESULTS AND DISCUSSION}

\section{Infrared Spectroscopy $(I R)$}

IR spectra of urethane prepolymer showed characteristic absorption bands at $1740 \mathrm{~cm}^{-1}$ and $3400 \mathrm{~cm}^{-1}$ corresponding to urethane and amide II ( $-\mathrm{NH}$ stretching), respectively. As the prepolymer is isocyanate terminated, an intense and sharp band due to NCO is observed at $2270 \mathrm{~cm}^{-1}$. IR spectra of IPNs showed all bands corresponding to urethane and methacrylate network and no additional bands thereby ruling out the possibility of any chemical interaction between the component networks.

\section{Mechanical Properties}

The tensile strength, elongation at break and Shore A hardness of homopolymers, and the corresponding IPNs are given in Table II. PMMA is characterized by highest 


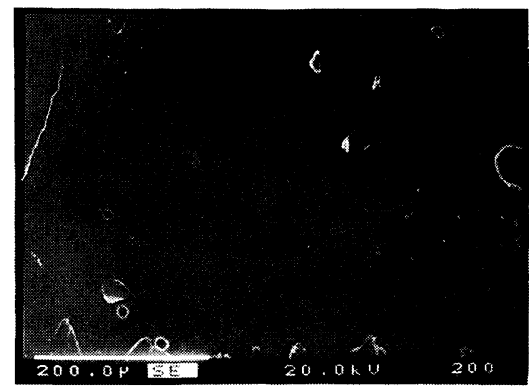

(a)

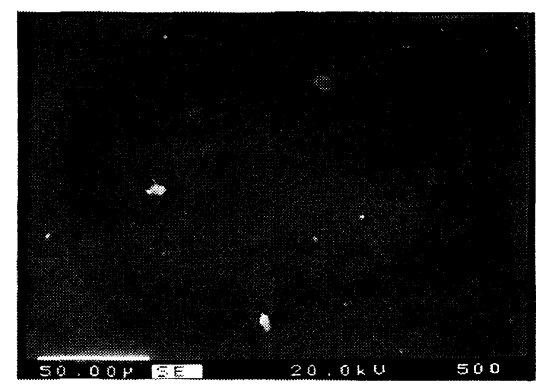

(b)

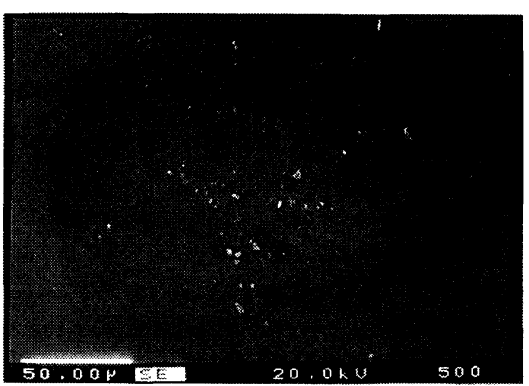

(c)

Figure 1. Representative Micrographs of (a) IPN-2, CU50/PMMA50, (b) IPN-5, CL1U50/PMMA50, and (c) IPN-8, CL2U50/PMMA50.

tensile strength and lowest elongation compared to urethane networks. It can be noted from Table II, that tensile strength decreased and elongation increased with increasing urethane content in the IPNs. In all the cases, variation of blend ratio resulted in drastic change in the elongation but only nominal change occurred in tensile strength. Thus, interpenetration of urethane and PMMA did not directly influence increase in the tensile strength of these IPN systems. Critical observation of Table II reveals that among all the IPNs unmodified castor oil urethane (CU)/PMMA IPNs exhibited relatively higher elongation, possibly due to the loosely packed structure, as observed from large domain size in CU/PMMA IPNs [Figure 1(a)] leading to increase in the free volume and mobility of elastomeric urethane chains. However, further inspection of Table II, reveals that ICL1U/ PMMA IPNs did not show enhancement in tensile strength and elongation over the parent network. The high degree of crosslinking in the ICL1U/PMMA IPN system probably lowers the statistical probability of threading (easy mobility of the entangled chains) thereby precluding the reinforcement of interpenetration. In all the IPNs except ICL1U/PMMA IPNs the composition having $75 \%$ urethane showed higher elongation than that of their parent networks, which can be ascribed to reinforcement of interpenetration.

Hardness data of IPNs (Table II) revealed that modified castor oil urethane/PMMA IPNs exhibited greater hardness than those from unmodified castor oil urethane/PMMA. This is because the fine domain size of ICL1U/PMMA and ICL2U/PMMA IPNs compared to CU/PMMA IPNs (Figure 1), indicated high degree of crosslinking density. Hardness increased with the concentration of PMMA in IPNs. Interestingly, ICL2U/ PMMA showed hardness greater than 100 Shore A at $75 \%$ concentration of PMMA. However hardness of CU/PMMA IPN's ranges between 70 to 92 Shore A.

\section{Apparent Density}

Actual and calculated densities based on volume additivity principle which states that $\left[d=w_{1} d_{1}+w_{2} d_{2}\right]$ where, $d$ is the density of the IPN sample, $w_{1}$ and $w_{2}$ are the weight fractions of the constituents, $d_{1}$ and $d_{2}$ are the corresponding densities, respectively, are listed in Table III. No definite trend can be seen from the density data. However, significant increase in density was observed with PMMA in ICL1U/PMMA and ICL2U/ PMMA IPNs, while this increase was less pronounced in CU/PMMA IPNs. This confirms that the degree of
Table III. Density of homopolymers and IPNs

\begin{tabular}{|c|c|c|c|}
\hline \multirow{2}{*}{$\begin{array}{l}\text { Sample } \\
\text { code }\end{array}$} & \multirow{2}{*}{$\begin{array}{c}\begin{array}{c}\text { Composition } \\
\text { U/PMMA }\end{array} \\
w t \%\end{array}$} & \multicolumn{2}{|c|}{ Density $/ \mathrm{g} \mathrm{cm}^{-3}$} \\
\hline & & Actual & Calculated $^{\mathrm{d}}$ \\
\hline $\mathrm{CU}^{\mathrm{a}}$ & $100 / 0$ & 1.08 & - \\
\hline IPN-1 & $25 / 75$ & 1.158 & 1.162 \\
\hline IPN-2 & $50 / 50$ & 1.124 & 1.136 \\
\hline IPN-3 & $75 / 25$ & 1.113 & 1.108 \\
\hline ICLIU $^{b}$ & $100 / 0$ & 1.036 & - \\
\hline IPN-4 & $25 / 75$ & 1.130 & 1.154 \\
\hline IPN-5 & $50 / 50$ & 1.080 & 1.114 \\
\hline IPN-6 & $75 / 25$ & 1.061 & 1.075 \\
\hline $\operatorname{ICL} 2 \mathbf{U}^{\mathrm{c}}$ & $100 / 0$ & 1.074 & - \\
\hline IPN-7 & $25 / 75$ & 1.158 & 1.163 \\
\hline IPN-8 & $50 / 50$ & 1.150 & 1.133 \\
\hline IPN-9 & $75 / 25$ & 1.062 & 1.104 \\
\hline PMMA & $0 / 100$ & 1.193 & - \\
\hline
\end{tabular}

Average error range: 0.011 .

${ }^{a} \mathrm{CU}=$ unmodified castor oil urethane. ${ }^{\mathrm{b}} \mathrm{ICL} 1 \mathrm{U}=$ modified castor oil (using 20\% linseed oil) urethane. ${ }^{\mathrm{c}}$ ICL2U $=$ modified castor oil (using $30 \%$ linseed oil) urethane. ${ }^{\mathrm{d}}$ Based on the volume additivity of the components.

interpenetration in the IPNs increases with modification of castor oil. Increase in the densities of the IPNs was observed due to the mixing of the component networks. This is attributed to the fine domain sizes of the ICL1U/PMMA and ICL2U/PMMA IPNs (Figure 1) which indicates excellent mixing and hence maximum interpenetration of urethane and PMMA. The maximum density of all IPNs is at $75 \%$ PMMA concentration. Comparing the densities of IPN it was observed that, the actual and calculated densities do not match with each other which implies that there seems to be a low density amorphous region at the interface between the two phases. It is the interpenetration that causes the low density amorphous region. The deviation between calculated and observed density is dependent on the amount of such an interpenetrating layer in the blend. ${ }^{17}$ Finally, it can be noted that densities of all the IPNs in each case lie between the densities of parent networks.

\section{Swelling Study}

Swelling experiments were carried out according to Sperling and Mihalakis ${ }^{16}$ and the percentage of swelling was calculated for each IPN according to the following equation: 
$\%$ Swelling $=$

(weight of swollen polymer - weight of dry polymer) $\times 100$

$\%$ swelling data of IPNs in MEK, toluene and water are presented in Table IV. It is evident from the table that no swelling occurred in water, whereas, swelling was more prominent in MEK and toluene. This is because of the ease of penetration of the solvents in to the core of the IPN matrix with less resistance, there by increasing swelling. In contrast to the gradual increase in swelling with increase in concentration of urethane in CU/PMMA IPNs, the swelling was highest at $50 \%$ urethane composition in ICL1U/PMMA and ICL2U/PMMA IPNs. Unmodified castor oil urethane and IPNs had relatively greater resistance to swellability than those synthesized from modified castor oil urethane and their IPNs. Swellability of IPNs was more prominent in toluene, due to the fact that toluene can penetrate the core of the IPN matrix with less resistance, thereby, increasing swelling.

\section{Morphology}

The morphology of IPNs is revealed by electron micrographs [Figures 1(a), 1(b), and 1(c)]. All IPNs showed microphase dispersion of PMMA phase in the

Table IV. Swelling of homopolymers and IPNs

\begin{tabular}{|c|c|c|c|c|}
\hline \multirow{2}{*}{$\begin{array}{l}\text { Sample } \\
\text { code }\end{array}$} & \multirow{2}{*}{$\begin{array}{c}\begin{array}{c}\text { Composition } \\
\text { U/PMMA }\end{array} \\
\frac{w t \%}{w} \%\end{array}$} & \multicolumn{3}{|c|}{ Swelling $/ \%$} \\
\hline & & Toluene & MEK & Water \\
\hline $\mathrm{CU}^{\mathrm{a}}$ & $100 / 0$ & 111 & 96 & 1 \\
\hline IPN-1 & $25 / 75$ & 103 & 98 & No change \\
\hline IPN-2 & $50 / 50$ & 118 & 103 & No change \\
\hline IPN-3 & $75 / 25$ & 121 & 111 & No change \\
\hline $\mathrm{ICLIU}^{\mathrm{b}}$ & $100 / 0$ & 124 & 105 & 1 \\
\hline IPN-4 & $25 / 75$ & 106 & 86 & No change \\
\hline IPN-5 & $50 / 50$ & 141 & 125 & No change \\
\hline IPN-6 & $75 / 25$ & 130 & 112 & No change \\
\hline ICL2U $U^{\mathrm{c}}$ & $100 / 0$ & 119 & 103 & 2 \\
\hline IPN-7 & $25 / 75$ & 104 & 99 & No change \\
\hline IPN-8 & $50 / 50$ & 128 & 114 & No change \\
\hline IPN-9 & $75 / 25$ & 116 & 106 & No change \\
\hline PMMA & $0 / 100$ & 96 & 101 & 1 \\
\hline
\end{tabular}

${ }^{\mathrm{a}} \mathrm{CU}=$ unmodified castor oil urethane. ${ }^{\mathrm{b}} \mathrm{ICL} 1 \mathrm{U}=$ modified castor oil (using 20\% linseed oil) urethane. ${ }^{\mathrm{c}}$ ICL2U = modified castor oil (using $30 \%$ linseed oil) urethane. urethane matrix. However, in case of IPN-8 (c), the domain size $(3 \mu \mathrm{m})$ is small and well mixed, whereas, in IPN-5 (b) phase domains are in the range of $3 \mu \mathrm{m}$ to $9 \mu \mathrm{m}$. Figure 1(a) shows that in IPN-2 along with microphase dispersion, large globules of the acrylate having domain sizes from $8 \mu \mathrm{m}$ to $30 \mu \mathrm{m}$ have also dispersed. This indicates higher compatibility of modified castor oil urethane/PMMA IPNs than those corresponding unmodified castor oil urethane/PMMA IPNs. It was interesting to note that the electron micrographs of IPN-2 when imaged at magnification above $200 \times$ and IPN-5 and IPN-8 at magnification above $500 \times$ develop cracks. The less resistance offered by the unmodified castor oil urethane/PMMA IPNs compared to their counterparts with the modified castor oil urethane/PMMA can be attributed to the softness of CU/PMMA IPNs which facilitates penetration of the imaged electron beam into the IPN film.

\section{Thermal Properties}

Thermal behavior of IPNs and homopolymers suggests that the thermal stability of the IPNs falls intermediate between those of urethane and PMMA. Clustering of the IPNs curves (Figure 2) indicates that there is no significant difference in degradation behavior. However, from the activation energy data (Table V) CU and the corresponding IPNs are relatively more stable than ICL1U, ICL2U, and their corresponding IPNs. In all the cases, generally, two stage decomposition is observed [Figures 3(a)-3(e)]. Stage I corresponds to the urethane bond breaking in case of uralkyd, whereas, in the IPNs,

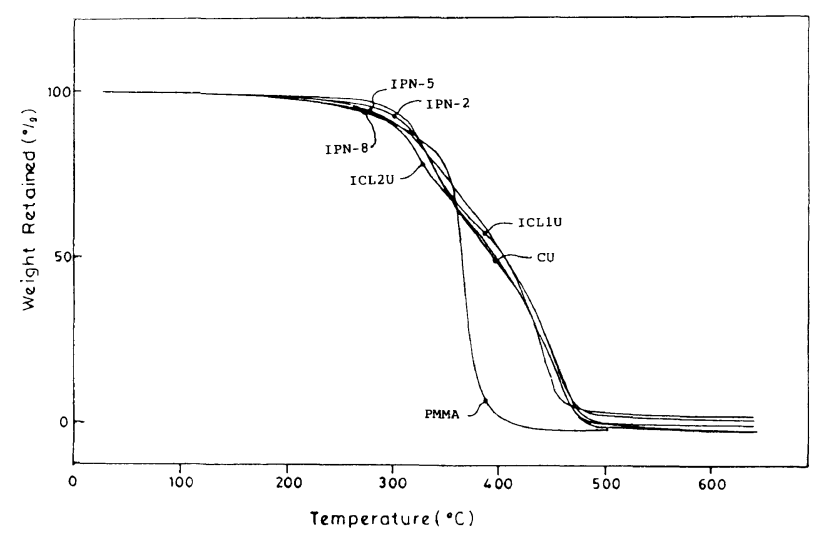

Figure 2. Representative thermograms for 50/50 (Urethane/PMMA) IPN, and corresponding homopolymers.

Table V. Thermal decomposition of homopolymers and IPNs

\begin{tabular}{|c|c|c|c|c|c|}
\hline \multirow{3}{*}{ Sample code } & \multirow{3}{*}{$\begin{array}{c}\text { Compositions } \\
\frac{\text { U/PMMA }}{\text { wt } \%}\end{array}$} & \multicolumn{4}{|c|}{ Decomposition stages } \\
\hline & & \multirow{2}{*}{ Stage $\mathrm{I} /{ }^{\circ} \mathrm{C}$} & Activation energy & \multirow{2}{*}{ Stage $\mathrm{II} /{ }^{\circ} \mathrm{C}$} & Activation energy \\
\hline & & & kcal mol${ }^{-1}$ & & $\mathrm{kcal} \mathrm{mol}^{-1}$ \\
\hline $\mathrm{CU}^{\mathrm{a}}$ & $100 / 0$ & $254-391$ & 36 & $391-502$ & 44 \\
\hline $\mathrm{ICL1}^{\mathrm{b}}$ & $100 / 0$ & $220-387$ & 32 & $387-502$ & 39 \\
\hline $\mathrm{ICL} \mathrm{U}^{\mathrm{c}}$ & $100 / 0$ & $185-353$ & 22 & $353-505$ & 34 \\
\hline IPN-2 & $50 / 50$ & $194-365$ & 29 & $365-562$ & 40 \\
\hline IPN-5 & $50 / 50$ & $182-378$ & 28 & $378-560$ & 36 \\
\hline IPN-8 & $50 / 50$ & $182-540$ & 17 & - & - \\
\hline PMMA & $0 / 100$ & $154-450$ & 16 & - & - \\
\hline
\end{tabular}

${ }^{\mathrm{a}} \mathrm{CU}=$ unmodified castor oil urethane. ${ }^{\mathrm{b}} \mathrm{ICL} 1 \mathrm{U}=$ modified castor oil (using $20 \%$ linseed oil) urethane. $\quad{ }^{\mathrm{c}} \mathrm{ICL} 2 \mathrm{U}=$ modified castor oil (using $30 \%$ linseed oil) urethane. 


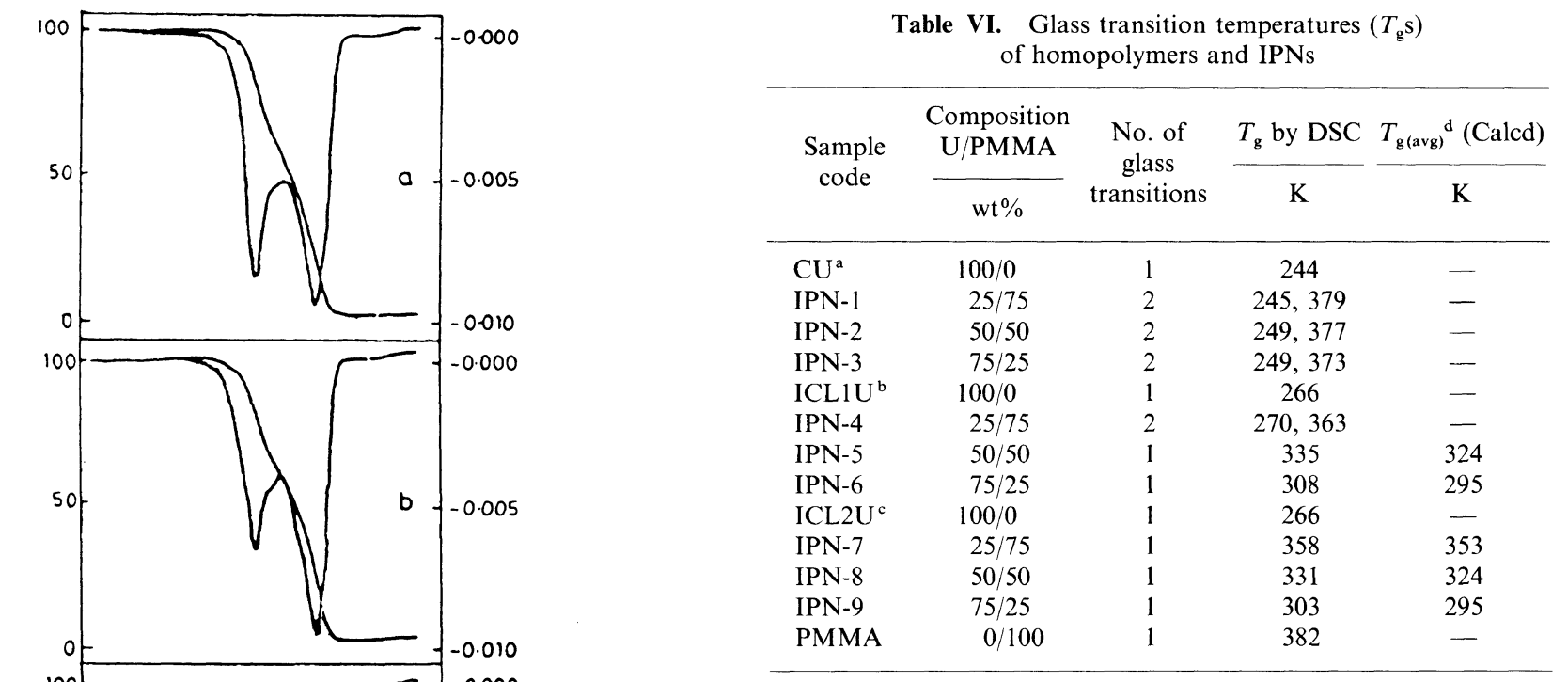

${ }^{a} \mathrm{CU}=$ unmodified castor oil urethane. ${ }^{\mathrm{b}} \mathrm{ICLIU}=$ modified castor oil (using 20\% linseed oil) urethane. ${ }^{\mathrm{c}}$ ICL2U $=$ modified castor oil (using $30 \%$ linseed oil) urethane. ${ }^{\mathrm{d}} T_{\mathrm{g}(\mathrm{avg})}=W_{1} T_{\mathrm{g} 1}+W_{2} T_{\mathrm{g} 2} ; W_{1}, W_{2}$ are the weight fractions of urethane and PMMA, respectively.

$500^{\circ} \mathrm{C}$ and IPNs at $550^{\circ} \mathrm{C}$. Interestingly, IPN-8 showed only one stage decomposition [Figure 3(f)]. This can be explained by the fact that the ICL2U and PMMA, are entangled in such a manner that during thermal degradation, the unzipped MMA monomer may be produced. This monomer might act as a free radical scavenger for PU degradation in the first stage delaying further the urethane bond breaking. ${ }^{19}$ The thermograms indicate that all IPNs decompose within 2-4\% weight in the temperature range of $0-200^{\circ} \mathrm{C}$, and about $10 \%$ weight loss occurred at $310-320^{\circ} \mathrm{C}$. There was rapid weight loss from $30-90 \%$ at $355-475^{\circ} \mathrm{C}$, because of decrosslinking of the two networks, and almost all IPNs decomposed completely from $475^{\circ} \mathrm{C}$ onward up to $550^{\circ} \mathrm{C}$. In this region along with the unzipped MMA monomer the polyol of the urethane network may possibly have become detached by a free radical mechanism. For all homopolymers and IPNs, activation energy values are lower in the first stage and higher in the second stage indicating that the degradation is relatively faster in the first stage than in second stage. TGA and derivative thermogram (DTG) of PMMA showed single stage decomposition and lower activation energy, indicating that the chain scissions of PMMA is the rapid process.

\section{Glass Transition Temperatures $\left(T_{\mathrm{g}} s\right)$}

Table VI shows that IPNs of CU/PMMA and ICL1U/PMMA (25/75) exhibit two glass transitions indicating phase separations. However, there is an inward shift of temperature corresponding to PU and PMMA phase indicating a small amount of polyurethane mixed with the polyacrylate phase. All other IPNs showed single glass transition intermediate between those of the parent networks. In case of ICL1U/PMMA and ICL2U/PMMA IPNs, the latter showed relatively less deviation of $T_{\mathrm{g}}$ from theoretically calculated $T_{\mathrm{g}}$ indicating high degree of interpenetration. It is difficult to correlate the composition and of IPNs with shifting in glass transition temperatures because of the wide distribution of phase domain and complexity of the morphology. along with urethane bond breaking, unzipping of PMMA network also takes place, in the temperature range $330-340^{\circ} \mathrm{C}$. Stage II, the polyol decomposition, occurs at $450-455^{\circ} \mathrm{C}$ for both, polyurethane (PU) and IPN. ${ }^{18}$ Finally, polyurethane showed complete weight loss at 


\section{CONCLUSIONS}

The introduction of more unsaturation in the castor oil enhances tensile strength, hardness of the polyurethane and its IPNs. No significant change was observed in swelling and thermal properties. Urethanes based on $30 \%$ linseed oil modified castor oil exhibits greater compatibility with PMMA followed by $20 \%$ linseed oil and unmodified castor oil. Thus, it can be concluded that modifying the castor oil using linseed oil makes it more plastic as desired rather than elastomeric.

\section{REFERENCES}

1. G. M.Yenwo, J. A. Manson, J. Pulido, L. H. Sperling, A. Conde, and N. Devia, J. Appl. Polym. Sci., 21, 1531 (1977).

2. L. H. Sperling, "Interpenetrating Polymer Networks and Related Materials," Plenum Press, New York, N.Y., 1981.

3. M. Patel and B. Suthar, J. Polym. Sci., Polym. Chem. Ed., 25, 2251 (1987).

4. P. Patel and B. Suthar, Polymer, 31, 339 (1990).
5. P. L. Nayak, S. Lenka, S. K. Panda, and T. Pattnaik, J. Appl. Polym. Sci., 47, 1089 (1993).

6. Chakrabarty and B. Das, J. Appl. Polym. Sci., 60, 2125 (1996).

7. P. Nayak, D. K. Mishra, D. Parida, K. C. Sahoo, M. Nanda, S. Lenka, and P. Nayak, J. Appl. Polym. Sci., 63, 671 (1997).

8. V. Athawale and S. Kolekar, Eur. Polym. J., in press.

9. G. C. Toon and G. S. Wooster, Official Digest, 32, 230 (1960).

10. J. Saunder and K. C. Frisch, "Polyurethanes: Chemistry and Technology," Part II, Interscience, New York, N.Y., 1964.

11. P. Das and Y. Nirvan, Paintindia, 44, 50 (1994).

12. O. S. Kabasakal, F. S. Guner, A. Arslan, A. Ergan, A. T. Erciyes, and Y. Yagci, J. Coating Technol., 68, 57 (1996).

13. A. O. C. S. Official Method, Cd 4-40 (1989).

14. H. L. Frisch and D. Klempner, Polym. Lett., 7, 775 (1969).

15. L. H. Sperling and E. N. Mihalakis, J. Appl. Polym. Sci., 17, 3811 (1973).

16. A. Broido, J. Polym. Sci., Part A-2, 7, 1761 (1969).

17. J. Quin, F. Li, Z. Wu, and B. Qian, in "Advances in Interpenetrating Polymer Networks," K. C. Frisch and D. Klempner, Ed., Vol. 2, Technomic, Lancaster, PA 1990, p 233.

18. P. D. Nair and M. Jayabalan, J. Polym. Sci., Polym. Chem. Ed., 28, 3775 (1990).

19. S. C. Klempner, D. Klempner, and K. C. Frisch, J. Appl. Polym. Sci., 21, 1289 (P977). 